\title{
Foreword to the proceedings of the Cemracs 2014
}

\author{
Martin Campos Pinto and Frédérique Charles
}

March 15, 2016

This volume contains contributions related to the CEMRACS 2014, which was devoted to the numerical modeling of plasmas.

The CEMRACS (Summer Mathematical Research Center on Scientific Computing and Applications, in french) is a 6-week scientific event that is organized every year in the CIRM, a CNRS mathematical institute located in the Calanques National Park near the city of Marseille, France. Under the auspices of the SMAI, its goal is to gather academic and industrial researchers to get an accurate focus on scientific computing problems that are relevant to both communities.

The 19th of the series, CEMRACS 2014 was devoted to the numerical modeling of plasmas, both in the kinetic and fluid regimes. It consisted of two consecutive events:

1. A summer school on the first week (July 21 - 25) with the following lectures.

- Fluid and transport modeling of plasmas given by James D. Callen (University of Wisconsin, US)

- Numerical simulations of large-scale structures in the Universe given by Stéphane Colombi (IAP - CNRS and UPMC, France)

- Modeling of Fusion Magnetized Plasmas given by Xavier Garbet (CEA - IRFM, France)

- Modeling for Inertial Confinement Fusion given by Laurent Masse (CEA - DIF, France)

- Confinement and Control in Plasmas given by Jean-Marcel Rax (Ecole polytechnique, France)

- Kinetic modeling and approximation given by Eric Sonnendrücker (IPP - TU München and MPI, Germany)

- Laser plasma interaction in high energy density physics given by Vladimir Tikhonchuk (CELIA - Bordeaux 1, France)

2. A 5-week research session (July 28 - August 29) dedicated to working in small groups, after a daily morning seminar.

The articles presented in this volume are the result of these research sessions, which gathered an average of 45 researchers every week (for a total of about 100 participants for the whole research session) working on 18 projects. In their majority, the articles study the performances of innovative schemes for plasma problems. Various numerical approaches are followed (such as multilevel finite volumes, optimal design processes, spline finite elements, semi-Lagrangian and particle methods, 
curvilinear and hexagonal meshes and model reduction techniques, to cite only a few) for problems posed either in fluid (e.g., MHD) or kinetic regimes. Some others, like the two first articles, slightly divert from the main course and conduct numerical studies of physical phenomenons in specific regimes (namely, the X-mode hybrid resonance in a magnetized plasma or the Maxwell-Boltzmann approximation for massless electrons in multispecies plasmas). Last but not least, a few articles address computer science issues such as proper development workflows or optimization of simulation codes (using particle methods, high-dimensional interpolations and gyroaverage computations) which are of specific interest for the numerical plasma community.

The research projects benefited from the support of many funding sources. In addition to the specific supports acknowledged in the proceedings, we would to express our gratitude to the HPC resources of the Aix-Marseille university and the Equip@Meso project, the ANR agency, the AMIES labex, the CEA, the CNPq in Brazil, the CNRS, the EM2C laboratory at Centrale Supeléc, the EFDA, EuroFusion, Euratom and the European Research Council, the HITEC graduate school, INRIA, the IRMIA Labex, the LJAD (Nice-Sophia Antipolis University) and LJLL (Paris 6 University) laboratories, the Maison de la Simulation in Saclay, the Max-Planck IPP and the TUM in Garching, Germany, and the SMAI.

We finally address our warmest thanks to the whole team at CIRM for their skills and spirit that allowed us to work during these research sessions in such a good mood, to our fellow organizers Boniface Nkonga and Hervé Guillard, to the colleagues from SMAI for their kind and efficient help on the technical matters (Olivier Goubet, Pauline Lafitte, Frédéric Lagoutière, Alain Prignet and Noura Sahtout), and to Pierre Navaro for his valuable expertise on the scientific computing issues.

And, of course to the participants of the summer school and the research projects, who happily spent part of their summer to make progresses on these useful problems. We can only wish the same pleasure for the organizers of the next CEMRACS sessions.

This is an Open Access article distributed under the terms of the Creative Commons Attribution License (http://creativecommons.org/licenses/by/4.0), which permits unrestricted use, distribution, and reproduction in any medium, provided the original work is properly cited. 